Journal of Information Systems Engineering \& Management

2019, 4(4), em0105

ISSN: $2468-4376$

\title{
Social CRM Analytics Challenges
}

\author{
Margarida Almeida Marques ${ }^{1 *}$, Carlos J. Costa ${ }^{1}$ \\ 1 ADV ANCE / CSG, ISEG (Lisbon School of Economics \& Management), Universidade de Lisboa, PORTUGAL
}

*Corresponding Author: margarida_228@live.com.pt

Citation: Marques, M. A. and Costa, C. J. (2019). Social CRM Analytics Challenges. Journal of Information Systems Engineering \& Management, 4(4), em0105. https:/ / doi.org/10.29333/jisem/ 6349

Published: December 7, 2019

\begin{abstract}
Social Customer Relationship Management (Social CRM) is an emerging concept that integrates traditional CRM and social networks, influenced by Web 2.0, to provide benefits for both organizations and customers. This paper aims to give a review of the existing literature on Social CRM, seeking later to develop a solution that helps to understand all the data that flow in a Social CRM system and that can help us to focus on a better strategy.
\end{abstract}

Keywords: Social CRM, social networks, CRM, Web 2.0, Business Analytics

\section{INTRODUCTION}

With the increasing evolution of the markets and the consequent stimulation of competition, it can be affirmed that there is currently an appreciation by organizations of relationship marketing. In this way, the management of recognized processes in the business world under the acronym CRM (Customer Relationship Management) becomes increasingly important for the stay and evolution of the effective and profitable organizations in the market.

The Social Network allows a new way of communication and interaction between companies and their clients, which changes the existing approach to customer relationship management (CRM) Baird and Parasnis (2013) and Kumar and Reinartz (2012). Within CRM, companies have only directional communication (for example, email) and collect information only on existing customers. Due to multi-directional communication through social networks, companies now have additional access to public and private information (e.g. profiles, activities, interests, etc.) of consumers (e.g. followers of the company's social network account), as well as like their respective "friends" Alt and Reinhold (2012). If on the one hand, CRM seeks to build strong and enduring relationships, on the other hand, comes the Web 2.0 that happens to cause a profound change to this concept. Web 2.0 tools are being implemented to improve relations with current and potential customers, making them feel, in addition to consumers, an integral part of the company as well as consumers. That is, the traditional Web model is being reversed, now it is the client who has the initiative to contact the companies.

The integration of social networks in CRM is a growing phenomenon in the research of Information Systems (SI), leading to a new scientific paradigm (Askool and Nakata, 2011) and is referred to as Social Customer Relationship Management (Social CRM). It is defined by Greenberg as "[...] a philosophy and a business strategy, supported by a technology platform, business rules, processes and social characteristics, designed to engage the customer in a collaborative conversation in order to provide mutually beneficial value in a trusted and transparent business environment, “. It corresponds to a philosophy and business strategy supported by a technology platform designed to engage the customer in a collaborative" conversation "that adds mutually beneficial value to a trusted and transparent business environment. 
Given this, the implementation of Social CRM requires efforts of transformation among all organizational parts. Nowadays, companies transform their businesses by applying new strategies, making organizational changes and buying new Social CRM technologies, thus achieving their competitive business benefits Greenberg (2010), Araujo, Costa and Aparicio (2017). However, there is still a lack of measurement tools for the performance and evaluation of Social CRM activities, as well as the achievement of the company's objectives. Thus, measuring the performance of social CRM is a challenge both in academic terms and in practice.

This article intends to investigate the impact of using Social CRM technology in an organizational perspective, focusing on the use of tools that help us interpret data from our website or from our social network. On the one hand, companies are still struggling to realize and evaluate the benefits of their implemented Social CRM technologies. On the other hand, the evidence on a corresponding approach to linking the use of Social CRM technology to the actual performance of Social CRM still lacks academic literature. Thus, the objective of this article is, after a literature review of these concepts, find a solution, a program / system, that helps us to obtain a statistical analysis of all the data we can achieve with our digital platforms that allow the company to develop their own marketing, for a future interpretation of them.

\section{THEORETICAL BACKGROUND}

\section{Web 2.0}

The use of the Internet as a space for business activity is not new territory (Costa, 1996). Within the period of Web 1.0, the Internet was understood as a means of unidirectional communication information. The content and the applications could only be created and published by individuals with technical and specific knowledge of software (Han, 2010). In contrast, Web 2.0 is described as the new generation of Internet users (Choudhury and Harrigan, 2014) and is characterized by the expansion of economic, social and technological trends that allow a new form of communication - the multidirectional one. Internet users are no longer exclusively information recipients, increasing the possibility of creating, modifying and exchanging their own content. Based on these characteristics, Web 2.0 can be defined as "the set of economic, social, and technological trends that collectively form the basis for the next generation of the Internet-a more mature, distinctive medium characterized by user participation, openness, and network effects "Lehmkuhl (2010). In fact, it is a set of economic, social and technological trends that together form the basis for the next generation of the Internet, a more mature and distinct medium characterized by user participation.

According to the creator of this concept, Tim O'Reilly with the article "What is Web 2.0?" (O'Reilly, 2005), there are seven principles of building Web 2.0 applications: the Web as a platform (example: free softwares ); collective intelligence (reception and creation of content); managing the database as a core competency (generating value for the content created); end of software update cycle (online beta versions); fast programming models, looking for simplicity in information architecture; software not limited to a single device, the computer is no longer the only possibility; user experience (active role of the user).

Figure 1 illustrates the one mentioned by the author (also presenting other notions of the Web 2.0 concept). With the advent of Web 2.0, companies have realized the potential of this new medium of communication. Traditional marketing, by which companies passed all their communication (magazines, television, newsletters and advertisements), is no longer enough to reach the market niches that companies seek. The digital age, revolutionized by the continuous evolution of Web 1.0 from Web 2.0 and the new interaction that results from its development, makes Marketing and business seek to find new ways to communicate with their target audience. 


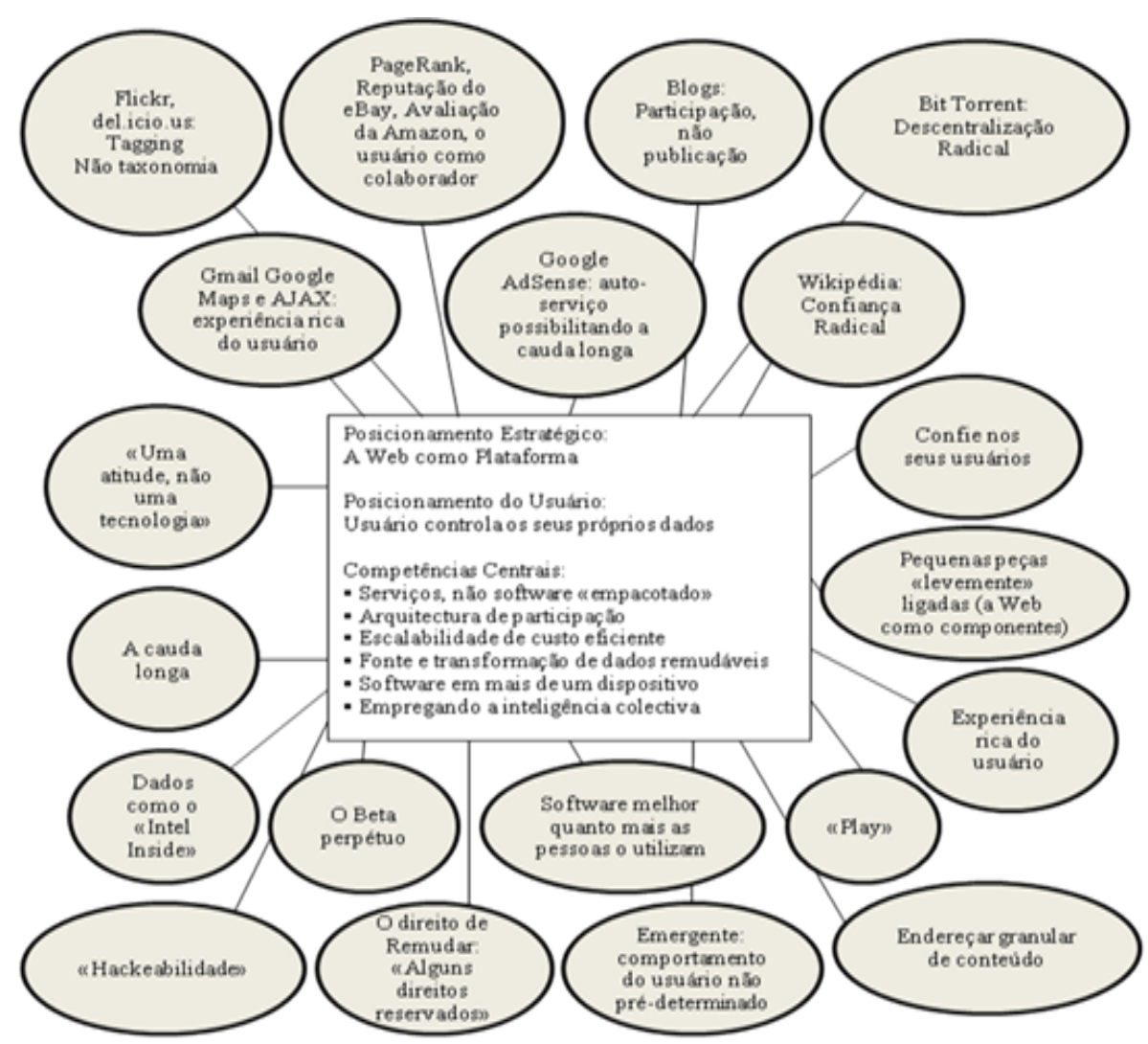

Figure 1. Web 2.0 Notions

\section{Social Media}

Social networks cannot be understood without first defining Web 2.0: a term that describes a new way in which end users use the World Wide Web, a place where content is continually changed by all operators in a collaborative way.

Social Networks includes a group of Internet-based applications, allowing the exchange of digital content. Features a front-end for the Internet user and allows Web 2.0 features. According to Kaplan and Haenlein (2010), these can be classified into six categories: social networking websites (for example, Facebook), blogs (including microblogs, for example Twitter), collaborative projects (eg Wikipedia), content (Youtube), virtual social worlds and worlds of virtual games (for example, World of Warcraft). The different functionalities recommended are, for example, relationships, such as Linkedin, where we can see how users are related to each other, groups, how to be part of a Facebook event, and shares, for example, in Instagram we can share photos. Having said all that, and according to, we can define social networks as a group of applications, based on the Internet and ideologically and technologically based by Web 2.0, which allow the creation and exchange of user generated content.

Sinclaire Jollean and Vougus (2019) cite O’Reilly's definition: "social media is a broad term that describes software tools that create user-generated content that can be shared", that is, the social network is a broad term describing tools software that creates user-generated content and can be shared. However, there are some basic requirements necessary for a website to be considered as a social network website: it must contain user profiles, content, a method that allows users to connect, post comments on each page and participation in virtual groups, based on their common interests (such as fashion or politics) (Costa and Alturas, 2010)

For many companies, the use of social networks to market their brand has been a great challenge. Ads, brochures and e-mails do not have the same impact as they did before. Companies must create new ways of impressing and differentiating themselves from the competition. (Costa and Alturas, 2010)

The use of collaborative systems and social networks by users and potential customers is something to be considered (Aparicio and Costa, 2012). What leads to its use can have a relevant impact to perceive users and potential customers (Costa and Aparicio, 2013). One thing is certain: using social networks, brands can create a strong social presence through online interaction with customers. Social tools can also help strengthen brand awareness by communicating core values, which in turn "opens" the opportunity to initiate conversations, develop partnerships, and expand the online community, conquering new followers and potential customers.

In a technology-driven world, social networking websites have become a medium where marketers can broaden their campaigns to a broader variety of consumers. To consider social networks as a marketing tool, we must take 
into account the previously mentioned concepts (Web 2.0 and Social Media). Chi (2011) defines social media marketing as a connection between brands and consumers, offering a personal channel for user-centric networks and social interaction. The tools and approaches to communicating with clients have changed a lot with the emergence of social networks, so companies must learn how to use social networks in a manner consistent with their business plan (Mangold and Faulds, 2009). This is particularly important for companies that strive to gain a competitive advantage.

One of the most characteristic traits of social networks and their users is their constant nature (Costa and Alturas, 2010). Companies, nowadays, should know that it is no longer credible to just tweet or refresh their Facebook page from time to time. Customers continuously crave novelties and innovations. Actively using social networking is one of the easiest ways to reach a large audience. Not only does an established network help you create new contacts, but it also helps you deepen your existing connections. Although this seems very familiar to traditional marketing techniques, social networks have given it a new "touch." Companies that fail to adopt a new, more interactive market will inevitably fall behind.

\section{CRM}

Consumers have evolved and are today more demanding, informed and autonomous. Technology has motivated this new profile and brands more than ever want to adapt to this reality. Customer Relationship Management (CRM) has emerged from the need for companies to build relationships with customers to meet their new demands. Behind it lies a marketing mix, centered on product and transactions, which is replaced by one that turns out to be more altruistic and puts the customer at the center of the universe (relational marketing). Lehmkuhl (2010) classifies the term relationship management in internal (employees, managers) and external relations (clients, government, suppliers): linking the second to the efforts made by marketers, we can describe the marketing relationship. CRM is, therefore, a subset of marketing relationships that refers exclusively to the customer. In the 1990s, CRM shifted from a transactional approach (focused on the company's products) to a relationship-based approach (focused on the customer relationship); however it was still a predominantly technological initiative, ignoring the important principles of marketing (Choudhury and Harrigan, 2014). Today, CRM is understood as a strategic relationship management and client-oriented approach (Kumar and W. Reinartz, 2012). The purpose of CRM is to establish the same approach in the company, which leads to long-term relationships with customers and the delivery of results to shareholders, which means a higher performance of the company. Thus, CRM can be defined as a strategic approach that is concerned with creating value for the customer (and for shareholders) by developing appropriate relationships with them. CRM thus unites the potential of marketing relationship strategies with IT to create profitable and lasting relationships with customers or other stakeholders.

\section{Social CRM}

With the advent of distributed nature embedded in Web 2.0 used in social networking technologies, CRM has a new opportunity to grow and thrive (Choudhury and Harrigan, 2014). The integration of social networks in CRM is a growing phenomenon, leading to a new scientific paradigm that is referred to as Social Customer Relationship Management (Social CRM). This, compared to traditional CRM, therefore uses the Web 2.0 concept to support the acquisition, maintenance and revitalization of the customer relationship and is described as a holistic, strategic and customer-centric approach (Lehmkuhl and Jung, 2013). These approaches to active listening, leads to encouragement and collection of information that may not stick to the purely commercial dimension, is what happens with crowdsourcing (Aparicio, Costa, and Braga, 2012). The relationship with the company ceases to be controlled by the company itself but based on the interactions between the company and the client, and between them and their friends or even other web users (such as consumers) (Choudhury and Harrigan, 2014). Within the Social CRM, the customer is then understood as a collaborative partner within a customer-centric environment (Greenberg, 2010), aiming at establishing a long-term relationship with the customer, in order to provide mutual beneficial value to the company and the customer (Choudhury and Harrigan, 2014). Social CRM does not replace traditional CRM, but it enriches existing management approaches. To conclude, Social CRM is defined as "a business philosophy and strategy, supported by a technology platform, processes and social characteristics, designed to engage the customer in a collaborative conversation to provide mutually beneficial value.

What happened was not a disaster of traditional CRM, but an optimization of this "revenue". That is, new features and tools have been associated with analyzing the consumer in social networks and talking to him (twoway conversation). If we know the consumer better and have more information about it in real time, we will be able to outline a more assertive strategy. Social CRM allows us to have a broad set of data that is subsequently translated into information and insights about the customer. Attentive to your needs and desires, you will be able to invest in the construction of a previously analyzed relationship. We know what interests you, what moves you and how best to engage you. The creation of content that satisfies the consumer can thus be optimized by the breadth of knowledge that technology allows. In the new chapter of marketing (content), Social CRM presents 


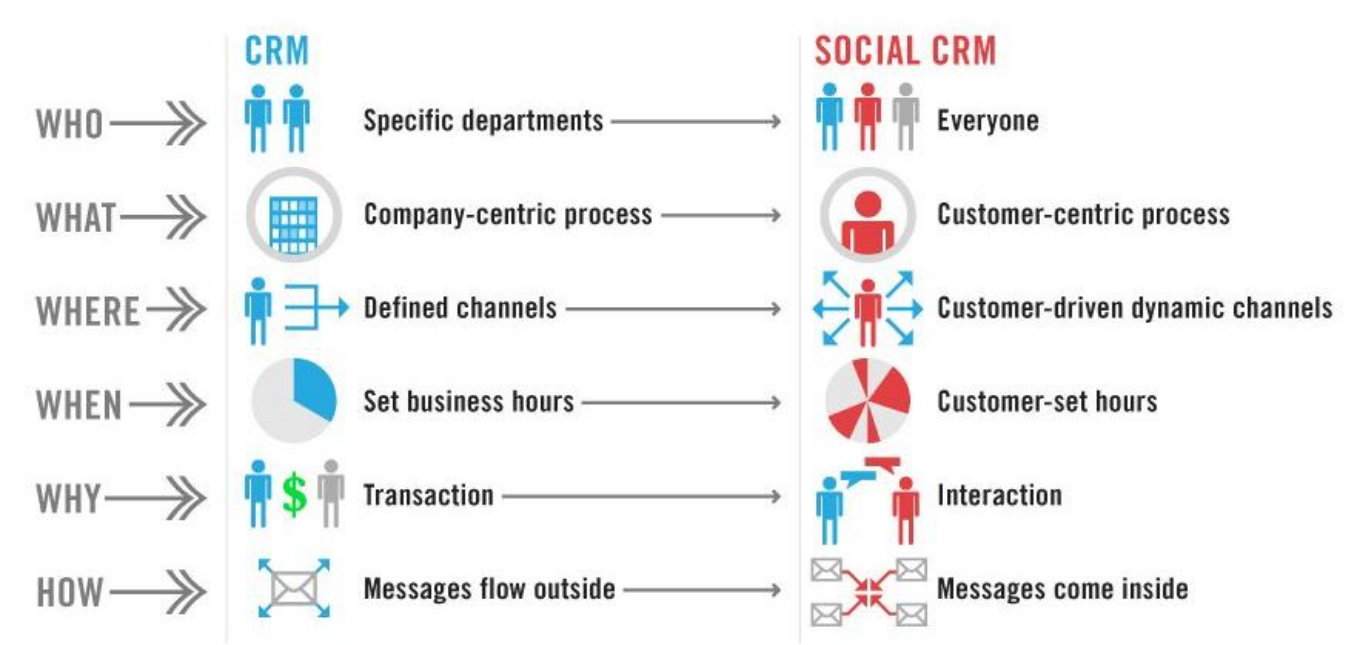

Figure 2. Differences between CRM and Social CRM (Source: Sexton, 2012)

Table 1. Social Media CRM

\begin{tabular}{lll}
\hline CRM Services & Category & Price \\
\hline Batchbook & Social CRM & Paid \\
\hline Commence CRM & CRM & Paid \\
\hline ConvergeHub & Social CRM & Paid \\
\hline Freshsales & Contact Management & Free \\
\hline HubSpot CRM & Social CRM & Free \\
\hline InStream & Online CRM & Paid \\
\hline Nutshell CRM & Social CRM & Paid \\
\hline PipelineDeals & Online CRM & Paid \\
\hline ProsperWorks & Lead Management & Paid \\
\hline Sailthru & Social CRM & Paid \\
\hline Salesboom CRM & CRM & Paid \\
\hline SalesforceIQ & Social CRM & Paid \\
\hline SalesLoft & Lead Management & Paid \\
\hline SalezShark & Online CRM & Paid \\
\hline Simple CRM System & Customer Service & Paid \\
\hline
\end{tabular}

itself as a privileged information tool and as the best strategic support for creating successful relationships between the customer and the brand. It is pure mathematics: more knowledge, better content, satisfied customer.

It is important to realize that a Social CRM developed with the help of a Big Data Analytics system can add automation to the tasks and anticipate the desires and needs of the customers. It is also important to highlight that the implementation of a CRM system requires a demanding back-up work. It is necessary to define, first and foremost, the objectives of technology integration, to understand the most significant advantages of this exercise and, above all, to understand that it is a huge cultural change in the organization. Many CRM projects fail precisely because this work of analysis and planning has not been effectively implemented. However, if the project is studied and implemented properly, there will certainly be substantial results optimization.

\section{TOOLS AND SELECTION}

Several tools may be used to implement social media approach. Among them, we may identify the following several categories, like Contact Management, lead management, Online CRM, customer service or Social CRM.

In Table 1, it is possible to identify some of the most popular services directly related to social CRM. A more detailed analyse will allow to identify many other services that although to being categorised as social CRM tools, may be used in the in this context. Some of those services are very related to specific social networks, like Pinterest Analytics, Twitter Analytics or Instagram Insights. Other are related to google services, like Google Alert or Google Analytics. Microsoft is also an important player with Microsoft Dynamics CRM. Socialmention is a very useful service with sentiment analysis implementation. This service is free, like Hubspot. But, the number of functionalities of the free version is very limited. To be useful, users must select paid version. The, other alternatives may be find, like Batchbook, Brand24, Brandwatch Analytics, Hootsuite, Nimble, Quintly, Salesforce, Sumall or Zoho. 
A superficial analysis allows to verify that there are many tools with specific purposes, and many characteristics. So, it is essential to select the most appropriate. To do it, must be employed a selection process. The selection process must follow a specific set of steps:

1. Identify main criteria, related to the functionalities needed

2. Identify weight of each criteria, according to the purpose

3. Identify possible solutions (software or service)

4. Wight each solution.

In want concern the main functionalities, it may include dashboard features, possibility of linking to specific social network service, sentiment analysis or statistic and datamining options.

Many of those tools already may be related to social analytics, social selling, voice of the customer, customer experience management. In what concerns data analysis, some of them are supported in unstructured data, using text mining, text analytics, natural language processing, sentiment analysis or machine learning (Wittwer, Reinhold, and Alt, 2016).

\section{CASE STUDY}

After we set up the Social CRM system and the data is already installed, what do we do with them now? And more importantly, how do we interpret them? This is where Google Analytics comes in to help us understand all the data flowing in a Social CRM system and can help us focus on a better strategy. Google Analytics is a traffic monitoring system that can be installed on any website, social network or blog. Its main goal is not only to know how many users access their platforms, but also how they behave when browsing the various pages and sections of the website. It's based on information gathered through Google Analytics that we can analyse whether visitors to a particular website are having the expected behaviour in response to a given online marketing campaign, doing exactly what it would expect, or if not, what could be made for the problem to be corrected. That is, Google Analytics not only lets you measure sales and conversions, but also provides you with current statistics on how visitors use your website, how they came to the website, and what to do to keep coming back. By accessing our account, we are directed to a registration form on the website. This form asks for basic data like address and name of our website, region, among others. Once completed, the tool provides an HTML code to include in all pages of the website (it's very simple, just copy and paste according to the instructions described next to the code). In case you are using a WordPress, installing a Plug-In and placing the mentioned code, it will also be possible to have Google Analytics installed on our website.

Google Analytics is not a crystal ball, that is, it does not point to where the problem is, it simply provides a lot of information from which to draw our conclusions (through graphs and generated tables). This is what we call Web Analytics, which, together with the tools mentioned, allows us to have a clear reading of all information regarding numbers and behaviors of users of any type of website.

Through the tools of Google Analytics, we can see some of the examples of analysis that we can derive from this system.

These tools allow us to explore our data, having standard reports that make it easier to measure and understand the interaction of the website. Plus, it takes just a few clicks to quickly create customized reports, visitor segments, and identify important data to share with the entire team. They let you see how many people are on the website right away, where they come from (country) and what they're viewing. With this analysis, we find out, for example, if the new content available is popular, whether the promotion of the day is driving traffic to our website and we can also see the immediate effects of our publications on social networks or blogs. The system allows defining the information to be analysed, creating a metrics panel itself, which facilitates, therefore, instead of searching the data, we have immediate access to the answers that are sought, offering the option of sharing, showing only the necessary information

Google analytics allows us to create and analyse our own customer segments by helping them understand and discover how closely they interact with the website. These variables allow us to customize Google Analytics and gather the data of the most important customers to the company. It allows us to know the path taken by the visitors to reach our website, offering several visualization tools. To facilitate its analysis, the system incorporates several graphs that present the information in the desired way. Content reporting helps identify the main content of the website and discover the "hidden treasures." In this way, we can know how often people visit each page of our platform, how long they stay in it, and how often they convert.

With this system it is possible to drive visitors to their destinations more quickly, and when they cannot find what they are looking for, they resort to searching. With reports from this tool, Google Analytics helps you find out what visitors are looking for in concrete by helping you identify missed product opportunities and speeding up the conversion process. Let's see how visitors move around on our website. When analysing reports, it is sometimes difficult to see how users navigate on a given page; using this tool we can therefore visually analyse its 
movement. It allows us to detect issues before our customers, which is very important, since we all know how frustrating the slowdown a website takes to load and how this can hurt both our advertising and search rankings. This tool makes it possible to analyse how long our pages take to load through a website speed report.

These reports help you measure the impact of social networks on your business goals and conversions, with integrated social and web data providing a global view of your content and community. The Conversions report allows you to quantify the value of social networks, assessing their impact. The Origins of Action in Social Networks report shows the ways social network visitors navigate to the Website, meaning that sources of action on social networks refer interested visitors. The Plug-Ins Social Networking report shows you the most shared articles on our website and the social buttons that are being clicked to share them (for example, the " +1 " button on Facebook or "Like" on Facebook). In addition, Activity Stream shows how visitors interact with your content on external websites, enabling us to find out what the visitors are sharing and where they are.

Those are some of the characteristics of the system that was used. But, some restrictions were also identified. In fact, when the case was being studied, EU parliament discussed several laws that may affect the used of CRM and Social Media. Among them, it is especially important General Data Protection Regulation (GDPR), he articles 13 and 11 of the Proposal for a Directive of the European Parliament and of the Council on copyright in the Digital Single Market.

The General Data Protection Regulation (GDPR) a regulation in European Union (EU) law on data protection and privacy for all individuals within the European Union and the European Economic Area (EEA). The GDPR aims primarily to give control to citizens and residents over their personal data and to simplify the regulatory environment for international business by unifying the regulation within the EU.

The EU has proposed a new directive on copyright. The article 13 of the Proposal for a Directive of the European Parliament and of the Council on copyright in the Digital Single Market is an attempt to reshape copyright law for the internet age. It regulates the relationship between copyright holders and online platforms, compelling the latter to enforce tighter regulation over protected content. According to this article, platform providers should "take measures to ensure the functioning of agreements concluded with rightsholders for the use of their works or other subject-matter or to prevent the availability on their services of works or other subjectmatter identified by rightsholders through the cooperation with the service providers." Those measures should be "appropriate and proportionate", and the platforms should provide rightsholders with "adequate reporting on the recognition and use of the works and other subject-matter". The article 13 can enable abusive behaviour, thereby threatening freedom of expression and information.

Article 11 introduces a "link tax", requiring that internet companies get permission from publishers to use a piece of their work. On websites like Google and Twitter, for instance, a small part of the article is usually shown before someone clicks into it entirely. But, under the new rule, those technology companies would have get permission and possibly even pay to use that excerpt.

\section{DISCUSSION}

Literature review allows to identify the potentialities of Social CRM. The case study also allowed to identify that the use of this type of tools have some challenges. The choice of the most adequate software or service is the first step. Then, users may react to the use of new software or services. Then, new laws may also be treats or opportunities to the use of Social CRM Analytics. New changes in the law related to intellectual property and data protection will probably contribute to clarification of some situations but also may contribute to restrict the access of some data.

\section{CONCLUSIONS}

The essential work reported in this article was translated in the literature review on the concept of Social CRM. As mentioned throughout the article, customer relationship management literature has long emphasized the need to create value as a means of developing and maintaining relationships with customers, achieved through the process of interaction with them and the respective management of the information obtained of these interactions. However, while CRM focuses on profitable and mutually beneficial development and maintenance, the results of the same initiatives have been mixed with many reported flaws. Marketers are often paying attention to social networking technologies as a means of overcoming the obstacles encountered in implementing CRM initiatives, extending it to a social CRM context. The problem developed from the need felt by the users, in this case the marketers, that is, the collection of analytical data with their traffic control on our website, has been solved through the tools provided by the Google Analytics program. 


\section{ACKNOWLEDGEMENT}

I gratefully acknowledge financial support from FCT- Fundação para a Ciencia e Tecnologia (Portugal), national funding through research grant UID/SOC/04521/2013.

\section{REFERENCES}

Aparicio M. and Costa, C. (2012). Collaborative systems: characteristics and features. In Proceedings of the 30th ACM international conference on Design of communication (SIGDOC '12). ACM, New York, NY, USA, https:/ / doi.org/141146 10.1145/2379057.2379087

Aparicio, M. Costa, C. and Simoes Braga. A. (2012). Proposing a system to support crowdsourcing. In Proceedings of the Workshop on Open Source and Design of Communication, ACM, pp. 13-17. https://doi.org/10.1145/2316936.2316940

Araujo, H., Costa, C. and Aparicio. M. (2017). Modelo de competitive intelligence (CI) competitive intelligence (CI) model. Information Systems and Technologies (CISTI), 2017 12th Iberian Conference on. IEEE https://doi.org/10.23919/CISTI.2017.7975787

Askool S. and Nakata, K. (2011) A conceptual model for acceptance of social CRM systems based on a scoping study. AI \& Society 26(3), 205-220. https:/ / doi.org/10.1007/s00146-010-0311-5

Baird, C. H. and Parasnis, G. (2013). From social media to Social CRM - What customers want, IBM.

Chi, H. (2011). Interactive Digital Advertising VS. Virtual Brand Community: Exploratory Study of User Motivation and Social Media Marketing Responses in Taiwan. Journal of Interactive Advertising, 12(1), 44-61. https://doi.org/10.1080/15252019.2011.10722190

Choudhury, M. and Harrigan, P. (2014). CRM to social CRM: the integration of new technologies into customer relationship management. Journal of Strategic Marketing, 22(2), 149-176. https:// doi.org/10.1080/0965254X.2013.876069

Costa, C. and Alturas, B. (2010). Social networks and design of communication. In Proceedings of the Workshop on Open Source and Design of Communication (OSDOC 10). ACM, New York, NY, USA, 11-14. https://doi.org/10.1145/1936755.1936759

Costa, C. and Aparicio, M. (2013). Social networks: intentions and usage. In Proceedings of the 2013 International Conference on Information Systems and Design of Communication (ISDOC '13). ACM, New York, NY, USA,. 101-107 https://doi.org/10.1145/2503859.2503875

Costa, C. (1996). Internet e Estratégia Empresarial. Revista Portuguesa de Marketing, 1(3), 88-97.

Greenberg, P. (2010). The impact of CRM 2.0 on customer insight. Journal of Business \& Industrial Marketing, 25(6), 410-419, https://doi.org/10.1108/08858621011066008

Han, S. (2010). Theorizing New Media: Reflexivity, Knowledge, and the Web 2.0. Sociological Inquiry 80.2, pp 200-213. https://doi.org/10.1111/j.1475-682X.2010.00327.x

Kaplan, A. and Haenlein, M. (2010). Users of the world, unite! The challenges and opportunities of Social Media. Business Horizons, 53, 59-68. https://doi.org/10.1016/j.bushor.2009.09.003

Kumar, V. and Reinartz, W. (2012). Customer Relationship Management. Springer Gabler, Berlin, Heidelberg,. https://doi.org/10.1007/978-3-662-55381-7

Lehmkuhl, T. and Jung, R. (2013). Towards Social CRM - Scoping the concept and guiding research. Proceedings of the 26th Bled e Conference.

Lehmkuhl, T. (2014). Towards Social CRM: A Model for Deploying Web 2.0 in Customer Relationship Management (PhD Dissertation), Bamberg: Difo-Druck GmbH.

Mangold, W. and Faulds, D. (2009). Social Media: The New Hybrid Element of the Promotion Mix. Business Horizons, 52(4), 357-365.

O'Reilly, T. (2005). What Is Web 2.0? http://www.oreillynet.com/pub/a/oreilly/tim/news/2005/09/30/whatis-web-20.html (Accessed December 2017)

Sexton, K (2012). Business Must Have: Social CRM Apps. http://www.business2community.com/mobileapps/business-must-have-social-crm-apps-0179264 (Accessed May 2015)

Sinclaire, J., Jollean, K. and Vogus, C. (2011). Adoption of social networking sites: an exploratory adaptive structuration perspective for global organizations. Information Technology and Management, 12(4), 293-314. https://doi.org/10.1007/s10799-011-0086-5

Wittwer, M, Reinhold, O. and Alt, R. (2016). Social Media Analytics in Social CRM-Towards a Research Agenda. BLED 2016 Proceedings. 32. https:// aisel.aisnet.org/bled2016/32 